ERRATUM

\title{
Lipid accumulation product: a powerful marker of metabolic syndrome in healthy population
}

Mariano J Taverna, María T Martínez-Larrad, Gustavo D Frechtel and Manuel Serrano-Ríos

The authors and the journal apologise for errors in Table 1 of the above article published in March 2011, vol 164, pp 559-567. Some data relating to women were inadvertently omitted from the table.

The clinical characteristics of the whole sample and women are published in full below.

Table 1 Clinical characteristics of the sample population.

\begin{tabular}{|c|c|c|c|c|c|}
\hline & \multirow[b]{2}{*}{$\begin{array}{c}\text { Whole sample } \\
\quad(n=768)\end{array}$} & \multicolumn{4}{|c|}{ Women $(n=416)$} \\
\hline & & $\begin{array}{c}\text { MS-NCEP + } \\
(n=64)\end{array}$ & $\begin{array}{c}\text { MS-NCEP- } \\
\quad(n=352)\end{array}$ & $\begin{array}{c}\text { MS-IDF+ } \\
(n=73)\end{array}$ & $\begin{array}{l}\text { MS-IDF- } \\
(n=343)\end{array}$ \\
\hline \multicolumn{6}{|c|}{ Continuous variables (mean \pm s.D.) } \\
\hline Age (years) & $54.0 \pm 11.6$ & $61.0 \pm 10.3$ & $53.3 \pm 11.3$ & $60.7 \pm 10.0$ & $53.1 \pm 11.4$ \\
\hline BMI $\left(\mathrm{kg} / \mathrm{m}^{2}\right)$ & $27.43 \pm 3.97$ & $31.84 \pm 4.04$ & $26.48 \pm 4.05$ & $31.22 \pm 4.19$ & $26.48 \pm 4.09$ \\
\hline WC $(\mathrm{cm})$ & $90.12 \pm 11.37$ & $98.05 \pm 8.21$ & $83.02 \pm 9.91$ & $96.34 \pm 9.00$ & $83.02 \pm 9.91$ \\
\hline $\mathrm{HC}(\mathrm{cm})$ & $100.23 \pm 7.96$ & $108.52 \pm 9.20$ & $100.19 \pm 7.99$ & $107.43 \pm 9.26$ & $100.20 \pm 8.05$ \\
\hline WHR & $0.90 \pm 0.10$ & $0.90 \pm 0.07$ & $0.83 \pm 0.07$ & $0.90 \pm 0.07$ & $0.83 \pm 0.07$ \\
\hline SBP $(\mathrm{mmHg})$ & $124.6 \pm 17.1$ & $140.21 \pm 16.99$ & $120.82 \pm 16.87$ & $140.33 \pm 16.35$ & $120.28 \pm 16.66$ \\
\hline $\mathrm{DBP}(\mathrm{mmHg})$ & $78.3 \pm 8.9$ & $85.03 \pm 9.04$ & $76.40 \pm 8.35$ & $84.62 \pm 9.02$ & $76.26 \pm 8.30$ \\
\hline $\mathrm{FPG}(\mathrm{mmol} / \mathrm{l})$ & $4.91 \pm 1.16$ & $5.76 \pm 1.88$ & $4.65 \pm 0.64$ & $5.72 \pm 1.81$ & $4.63 \pm 0.61$ \\
\hline $2 \mathrm{~h}$ glucose $(\mathrm{mmol} / \mathrm{l})$ & $6.43 \pm 2.39$ & $8.55 \pm 3.00$ & $6.04 \pm 1.94$ & $8.49 \pm 2.93$ & $6.00 \pm 1.91$ \\
\hline Fasting insulin $(\mu \mathrm{U} / \mathrm{ml})$ & $13.21 \pm 8.65$ & $22.38 \pm 15.32$ & $11.31 \pm 6.61$ & $21.24 \pm 14.88$ & $11.29 \pm 6.65$ \\
\hline $2 \mathrm{~h}$ insulin $(\mu \mathrm{U} / \mathrm{ml})$ & $76.41 \pm 66.16$ & $130.57 \pm 92.67$ & $69.42 \pm 54.87$ & $122.08 \pm 90.46$ & $69.66 \pm 55.32$ \\
\hline Fasting proinsulin (pmol/l) & $10.67 \pm 8.37$ & $15.96 \pm 11.18$ & $8.45 \pm 5.70$ & $15.07 \pm 10.91$ & $8.46 \pm 5.73$ \\
\hline HOMA-IR & $1.76 \pm 0.95$ & $2.70 \pm 1.30$ & $1.53 \pm 0.73$ & $2.57 \pm 1.29$ & $1.52 \pm 0.73$ \\
\hline $\mathrm{TC}(\mathrm{mg} / \mathrm{dl})$ & $213.69 \pm 39.19$ & $218.67 \pm 46.08$ & $210.49 \pm 37.61$ & $218.42 \pm 47.51$ & $210.32 \pm 36.97$ \\
\hline LDL-C (mg/dl) & $133.20 \pm 34.28$ & $140.06 \pm 36.18$ & $127.64 \pm 32.67$ & $139.92 \pm 36.77$ & $127.34 \pm 32.38$ \\
\hline $\mathrm{HDL}-\mathrm{C}(\mathrm{mg} / \mathrm{dl})$ & $60.18 \pm 16.39$ & $52.89 \pm 12.71$ & $66.64 \pm 16.48$ & $53.27 \pm 13.30$ & $66.93 \pm 16.38$ \\
\hline $\mathrm{TG}(\mathrm{mg} / \mathrm{dl})$ & $98.12 \pm 60.37$ & $128.63 \pm 57.84$ & $73.78 \pm 29.59$ & $126.18 \pm 57.13$ & $72.87 \pm 28.36$ \\
\hline TG/HDL-C ratio & $1.90 \pm 1.74$ & $2.61 \pm 1.40$ & $1.21 \pm 0.71$ & $2.53 \pm 1.36$ & $1.18 \pm 0.69$ \\
\hline LAP & $34.65 \pm 28.74$ & $58.79 \pm 30.00$ & $21.96 \pm 14.17$ & $55.50 \pm 29.85$ & $21.70 \pm 14.07$ \\
\hline \multicolumn{6}{|l|}{ Dichotomic variables (\%) } \\
\hline Sedentary & 33.1 & 34.4 & 27.8 & 31.5 & 28.3 \\
\hline Smoking & 22.7 & 9.4 & 16.5 & 8.2 & 16.9 \\
\hline Obesity & 24.8 & 64.1 & 19.6 & 56.2 & 20.1 \\
\hline $\mathrm{HW}$ & 10.8 & 35.9 & 2.6 & 32.9 & 2.3 \\
\hline EWETS & 14.2 & 39.1 & 2.3 & 34.2 & 2.3 \\
\hline $\mathrm{FPG} \geq 5.6 \mathrm{mmol} / \mathrm{l}$ & 15.1 & 54.7 & 4.0 & 53.4 & 2.9 \\
\hline $\mathrm{TG} \geq 150 \mathrm{mg} / \mathrm{dl}$ & 13.5 & 31.3 & 1.7 & 30.1 & 1.2 \\
\hline Low HDL-C & 14.3 & 50.0 & 11.1 & 47.9 & 10.5 \\
\hline High $\mathrm{BP}^{\mathrm{b}}$ & 41.7 & 85.9 & 30.9 & 87.7 & 29.1 \\
\hline High WC (NCEP/ATP III) ${ }^{c}$ & 32.0 & 98.4 & 30.1 & 86.3 & 30.9 \\
\hline High WC (IDF) ${ }^{\mathrm{d}}$ & 63.7 & 100.0 & 51.1 & 100.0 & 58.6 \\
\hline MŠ-NCEP/ATP III & 15.2 & NA & NA & 87.7 & 0.0 \\
\hline MS-IDF & 18.9 & 100.0 & 2.6 & NA & NA \\
\hline
\end{tabular}

BMI, body mass index; BP, blood pressure; DBP, diastolic blood pressure; EWETS, enlarged waist elevated triglyceride syndrome; FPG, fasting plasma glucose; HC, hip circumference; HDL-C, high-density lipoprotein cholesterol; HOMA-IR, homeostasis model assessment of insulin resistance; HW, hypertriglyceridemic waist; LAP, lipid accumulation product; LDL-C, low-density lipoprotein cholesterol; NA, non applicable; SBP, systolic blood pressure; MS-IDF, International Diabetes Federation-diagnosed metabolic syndrome; MS-NCEP/ATP III, National Cholesterol Education Program/Adult Treatment Panel III-diagnosed metabolic syndrome; TC, total cholesterol; TG, triglycerides; TG/HDL-C ratio, triglycerides-to-high-density lipoprotein cholesterol ratio; WC, waist circumference; WHR, waist-to-hip circumference.

${ }^{a} \mathrm{HDL}-\mathrm{C}<40 \mathrm{mg} / \mathrm{dl}$ (men) or $<50 \mathrm{mg} / \mathrm{dl}$ (women).

${ }^{b} S B P \geq 130$ and/or DBP $\geq 85 \mathrm{mmHg}$ or treatment of diagnosed hypertension.

cWC $>102 \mathrm{~cm}$ (men) or $>88 \mathrm{~cm}$ (women).

${ }^{d} W C \geq 94 \mathrm{~cm}$ (men) or $\geq 80 \mathrm{~cm}$ (women). 